\title{
ACTION DE L'URÉE SUR LES SÉCRÉTIONS MAMMAIRES DE LA VACHE (') \\ IAR
}

\section{S. Z. ZELTER et G. CHARLET-LERY ( ${ }^{(2)}$.}

Laboratoire de Recherches zootechniques, Institut national Agronomique, Paris.

Les récentes revties publiées par REID (I) et par F'ERR.iNdo (2) témoignent du nombre considérable de travaux effectués sur l'utilisation de l'azote non différencié par les ruminants. En dépit des multiples expériences entreprises sur l'urée, qui a été expérimentée pour la première fois sur vaches laitières en I922 par Wol,TZ et coll. (3) et par RICHARDSEN et BRINKMANN (4), l'efficacité de l'azote uréique pour la sécrétion de lait de vache est encore fortement controversée. Malgré des résultats expérimentaux relativement similaires, les opinions formulées dans la dernière décade sont contradictoires: à l'efficacité équivalente à celle d'une farine de sang trouvée par Owen (5), de tourteaux usuels (lin, soja, coton), signalée pat RUT'EI, (6), WiLLETT (7), Hastings (8) et Thompson (9), on peut opposer les données considérées comme négatives par ARChibald (IO) et surtout par BARTLETT et BLAX'TER (II) qui, ayant entrepris une expérience massale, pensent non seulement que l'urée est dépourvue de toute action sur la production de lait, mais qu'elle serait même susceptible d'exercer à forte dose un effet dépressif.

Avec ces contradictions, il faut relever le fait qu'en France les rations habituellement distribuées aux vaches laitières diffèrent nettement (fourrages, racines et tubercules très riches en azote non protéique) de celles employées couramment aux États-Unis (prédominance de mélanges concentrés contenant en abondance de l'azote différencié) où des résultats positifs sont à nouveau signalés avec l'urée. Nous nous sommes donc posé la question de savoir ( $\left.{ }^{3}\right)$ si, dans les conditions normales d'élevage qui prévalent dans notre pays, l'azote uréique pouvait remplacer

(1) Avec le concours financier de l'Office National Industriel de l'Azote.

(2) Avec la collaboration technique de B. Dupont.

(3) Communication au $4^{\mathbf{e}}$ Congrès international de nutrition, Paris, Juillet $195 \%, \mathbf{1}^{3} .31$. 
avantageusement les sources azotées habituelles de supplémentation (tourteaux de lin et d'arachide). Nous avons dans ce dessein observé, comparativement à ces tourteaux, l'incidence sur la production de lait et de graisse de lait, d'une incorporation ou d'une suppression non compensée d'urée. Les mesures expérimentales ont débuté après une longue phase de préparation des animaux, le I er février 1956 pour se terminer 86 jours après.

\section{TECHNIQUE EXPÉRIMENTAIE}

\section{A. - RÉGIMES ALIMENTAIRES}

Un régime de référence normalisé a été adopté; il comportait pour tous les sujets:

Io Une partie quantitativement fixe composée de:

- betteraves fourragères ( 36 à $38 \mathrm{~kg}$ ) correspondant à un apport journalier d'environ $4 \mathrm{~kg}$ de matière sèche (excepté la période de préexpérience durant laquelle cette quantité n'a été en moyenne que de $2,8 \mathrm{~kg}$ par suite du gel intense qui a empêché la distribution régulière de racines;

- balles de blé (selon les périodes 4,6 à $5,6 \mathrm{~kg}$ ) ;

- foin de pré $(5 \mathrm{~kg})$ et foin de luzerne $(2,5 \mathrm{~kg})$; ce dernier a été remplacé lors de la post-expérience par du foin de minette (medicago lupulina) de valeur énergétique et azotée équivalente.

$2^{\circ}$ Une partie complémentaire quantitativement variable de manière à satisfaire les dépenses d'énergie et d'azote correspondant aux besoins individuels d'entretien et de production. Elle était constituée de 2 mélanges concentrés:

- mélange céréalier C (avoine $36 \%$, orge $43 \%$, son $2 \mathrm{I} \%$ ) pauvre en azote ( $\mathrm{I}, \mathrm{IO} \%$ de $\mathrm{N}$ Kjeldahl digestible) ;

- mélange de tourteaux- $\mathrm{T}$ (tourteau de $\operatorname{lin} 67 \%$, tourteau arachide $33 \%)$ riche en azote $(5,72 \%$ de $\mathrm{N}$ Kjeldahl digestible). Dans certains cas, ce dernier mélange a été remplacé par du tourteau d'arachide pur ( $7,9^{8 \%}$ de $\mathrm{N} \mathrm{Kjeldah1} \mathrm{digestible).}$

250 grammes de composé minéral (poudre d'os $40 \%$, ClNa $30 \%$, $\mathrm{Ca} \mathrm{CO}_{3} 20 \%, \mathrm{SO}_{4} \mathrm{Mg}$ Io $\%$ ) complétaient chaque ration journalière individuelle.

Le régime d'expérience différait de celui de référence par:

- soit la substitution d'urée aux tourteaux de manière à ne pas modifier le taux d'azote (expérience I). I,es quantités distribuées par vache et par jour oscillaient entre 98 et $193 \mathrm{~g}$ suivant le niveau individuel de production laitière, ce qui correspondait à une absorption de 0, I7 à $0,30 \mathrm{~g}$ d'urée par $\mathrm{kg}$ de poids vif. Ces doses ont été données en 
partie avec le repas de betteraves et en partie avec le mélange céréalier ;

- soit la suppression, non compensée, toutes conditions égales d'ailleurs, de l'azote uréique (expérience II) dans un but de contreépreuve.

Des prélèvements systématiques d'aliments ont été soumis à l'analyse chimique selon les techniques usuelles afin d'en connaître la composition et d'en déterminer la valeur nutritive.

\section{B. - MATÉRIEL ANIMAL ET DISPOSITIF EXPÉRIMENTAL}

Douze sujets d'expérience ont été choisis dans une étable de vaches normandes dont le comportement a été observé pendant près de six semaines dans des conditions similaires d'alimentation et d'environnement, après ajustement convenable de leur niveau de nutrition individuel à l'aide du régime qui devait, par la suite, servir de régime témoin. A l'issue d'une période de préexpérience qui a succédé à cette étape préparatoire, les douze animaux, pris après la phase ascendante de leur lactation, ont été répartis en deux groupes comparables de six sujets. Un tirage au sort a désigné l'un d'eux comme témoin (groupe A), l'autre (groupe B) étant utilisé pour le testage de l'urée.

I a technique combinée de groupes comparables et de périodes successives a été appliquée ; 4 à 5 jours de transition séparaient 2 périodes successives.

L'essai s'est déroulé selon le schéma suivant:

\begin{tabular}{|c|c|c|c|c|}
\hline $\begin{array}{c}\text { Période } \\
\text { Jurée en jours }\end{array}$ & $\begin{array}{c}\text { Pré-expérience } \\
(a) \\
2 \mathrm{I}\end{array}$ & $\begin{array}{l}\text { Explérience I } \\
\quad(b) \\
\mathbf{2 I}\end{array}$ & $\begin{array}{l}\text { Expérience II } \\
\qquad(c) \\
2 \mathrm{I}\end{array}$ & $\begin{array}{c}\text { Post-expérience } \\
(d) \\
\text { I5 }\end{array}$ \\
\hline Groupe A.. & \multicolumn{4}{|c|}{ Régime de référence (Tourteaux + Céréales) } \\
\hline Groupe B... & $\begin{array}{l}\text { Régime de } \\
\text { référence } \\
\text { ('Tourteaux } \\
+ \text { Céréales) }\end{array}$ & $\begin{array}{c}\text { Régime } \\
\text { d'expoérience } \\
\text { (Urée + Céréales) }\end{array}$ & $\begin{array}{c}\text { Régime } \\
\text { d'expérience } \\
\text { (Céréales } \\
\text { seulement) }\end{array}$ & $\begin{array}{l}\text { Régime de } \\
\text { référence } \\
\text { (Tourteaux } \\
+ \text { Céréales) }\end{array}$ \\
\hline
\end{tabular}

\section{C. - MESURES EXPÉRIMENTALES}

\section{$I^{0}$ Contrôle des consommations.}

Les rations étaient pesées journellement et ajustées pour chaque sujet tous les cinq jours, compte tenu de l'évolution de sa production 
laitière et lipidique et de son poids vif. Les refus étaient défalqués des rations distribuées.

\section{$2^{\circ}$ Contrôle des sécrétions lactée et lipidique.}

Au cours des deux traites effectuées journellement, les laits individuels produits étaient pesés. Leur richesse lipidique était dosée quotidiennement par la méthode Gerber sur un échantillon proportionnel aux quantités récoltées à chaque traite, et la matière grasse totale sécrétée était calculée en conséquence.

\section{$3^{\circ}$ Contrôle des variations de poids corporel.}

Des pesées individuelles étaient exécutées dans des conditions identiques pendant trois jours consécutifs à l'entrée et à l'issue de chaque période et hebdomadairement au cours de chacune d'elles. La variation journalière moyenne du poids de chaque individu a été déterminée au moyen de la droite de régression calculée à partir des données périodiques ainsi recueillies.

\section{RÉSULTATS}

Nos mesures ont été en partie enregistrées par un froid extrêmement intense et prolongé, auquel les animaux ont réagi par une diminution brutale de leur production, particulièrement marquée au cours de la préexpérience (voir fig. I et 2), qui coïncide avec l'installation brusque de températures anormalement basses. Il n'est pas exclu que ces réactions aient interféré avec celles dues aux traitements et masqué, partiellement du moins, la téponse des vaches à ceux-ci.

\section{A. - CONSOMMATION DE NOURRITURE}

Il n'a pas été constaté d'inappétence ou d'intolérance du fait de la présence d'urée dans le régime.

Les consommations moyennes d'éléments nutritifs (refus déduits) sont consignées dans le tableau I qui appelle quelques commentaires.

- les rations distribuées devaient, conformément aux normes françaises, pourvoir aux besoins d'entretien en fonction du poids vif et assurer 0,38 u. f. par kg de lait à $4 \%$ de graisse; durant la préexpérience, les ingesta ne couvraient pas la totalité des besoins théoriques, en raison de l'impossibilité de ravitailler régulièrement les animaux 


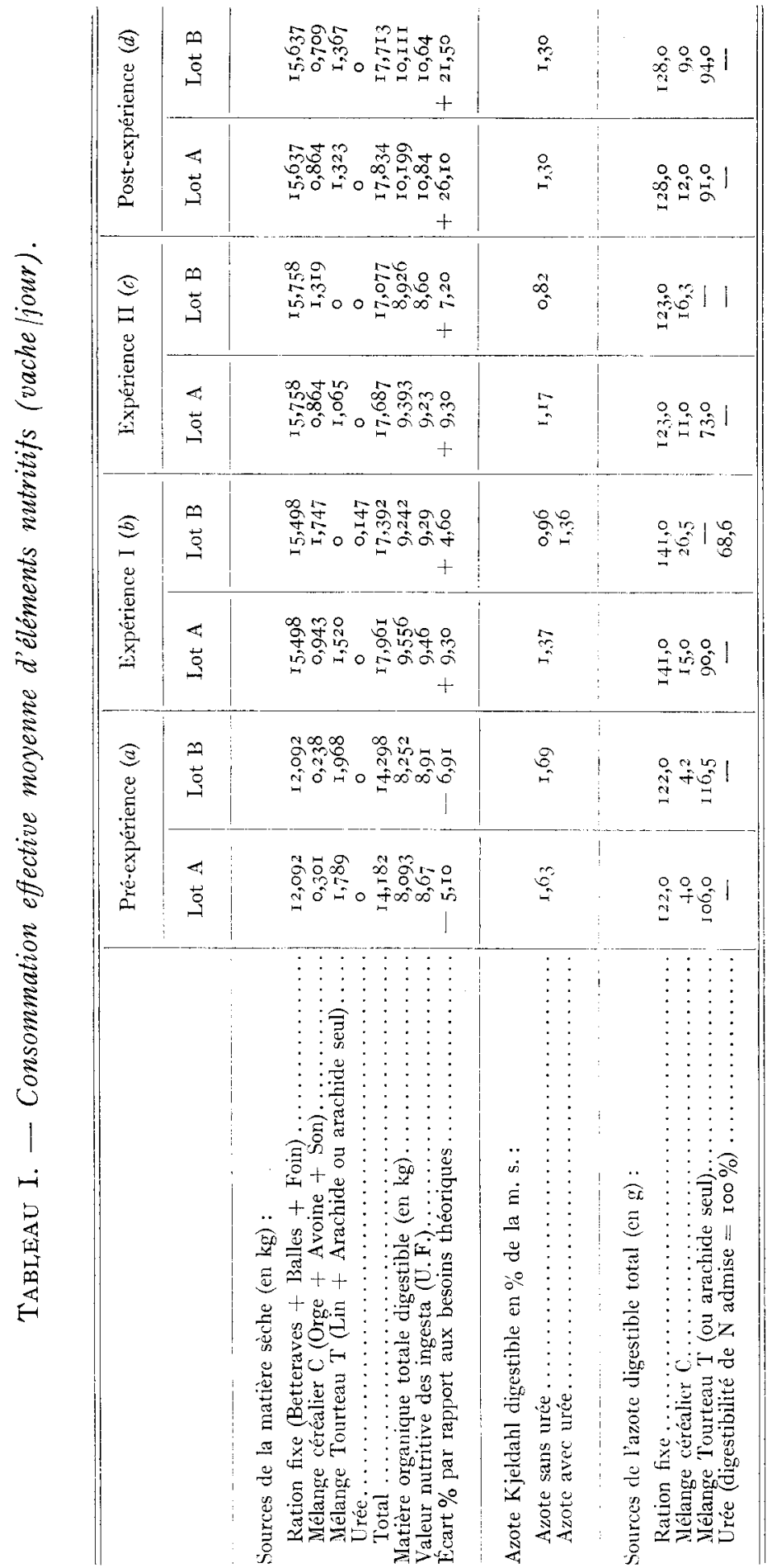


en betteraves, par suite des intempéries; pendant toutes les autres périodes, les apports dépassaient largement ces besoins; le déficit ou l'excédent d'énergie était comparable pour les 2 groupes (tabl. I) ;

- dans l'ensemble, les régimes étaient légèrement insuffisants en graisses digestibles; durant les pré-et post-expériences, tous les sujets en ingéraient quotidiennement 240 à $25 \mathrm{I} \mathrm{g}$; pendant 1'expérience I, les témoins en recevaient 226 et ceux traités à l'urée IgI, doses qui se sont abaissées respectivement à 207 et I77 g au cours de l'expérience II ; mais, étant donné la ration abondante de fourrages secs relativement cellulosiques, l'acétogénèse à partir de ces aliments a dî suppléer sans difficulté à une éventuelle carence lipidique (I2) ;

- les taux azotés étaient similaires dans les deux groupes lorsque ceux-ci consommaient le régime de référence (pré- et post-expérience) ; ils étaient, à priori, excédentaires par rapport aux besoins. Compte tenu de l'apport de l'urée, ils sont restés comparables pendant l'expérience I (période b) pour les deux lots; si l'on néglige cet apport, le niveau protidique des sujets du groupe expérimental ne représente que $70 \%$ de celui du témoin. Le groupe expérimental a été soumis intentionnellement à ce niveau, subnormal, au cours de la contre-épreuve (expérience II) où l'urée a été supprimée sans contrepartie, afin de pouvoir mieux déceler l'éventualité de l'utilisation de l'azote uréique pour des besoins de lactation.

Quant à la répartition de l'azote ingéré, en fonction de la source, le tableau II montre que les fourrages grossiers et aqueux (betterave, balle, foin) très riches en azote non différencié, ont fourni à eux seuls de 50,3 à $59,7 \%$ de l'azote total digestible ingéré; sauf au cours de la période qui servait de contre-expérience où cette fraction en a procuré au lot expérimental $88,3 \%$.

'TABLEAU II

Répartition de l'azote total digestible de la ration

en fonction de sa source.

\begin{tabular}{|c|c|c|c|c|c|c|c|c|}
\hline \multirow[b]{2}{*}{ Source azotée } & \multicolumn{2}{|c|}{ Pré-expérience } & \multicolumn{2}{|c|}{ Expérience I } & \multicolumn{2}{|c|}{ Expérience II } & \multicolumn{2}{|c|}{ Post-expérience } \\
\hline & $\begin{array}{l}\text { Témoin } \\
(A)\end{array}$ & $\begin{array}{l}\text { Expérim }{ }^{1} \\
\text { (B) }\end{array}$ & $\begin{array}{l}\text { Témoin } \\
\text { (A) }\end{array}$ & $\mid \begin{array}{c}\text { Expérim } 1 \\
\text { (B) }\end{array}$ & $\begin{array}{l}\text { Témoin } \\
(A)\end{array}$ & $\begin{array}{c}\text { Expérim } 1 \\
\text { (B) }\end{array}$ & $\begin{array}{l}\text { Témoin } \\
\text { (A) }\end{array}$ & $\underset{\text { Expérim }}{ }{ }^{1}$ \\
\hline $\begin{array}{c}\text { Ration fixe (bet- } \\
\text { teraves, foins, } \\
\text { balles)....... }\end{array}$ & & & & & & & & \\
\hline Mélange céréalier & $\begin{array}{r}32,0 \\
1,7\end{array}$ & $\Upsilon, 7$ & $6, \mathrm{I}$ & I I, & $\begin{array}{r}59, \\
5,\end{array}$ & $\begin{array}{l}00,3 \\
\mathrm{I} x, 7\end{array}$ & $\begin{array}{r}55,4 \\
5,2\end{array}$ & $\begin{array}{r}55,4 \\
3,9\end{array}$ \\
\hline Tourte & 45,7 & 48,0 & 36,6 & 0,0 & 35,3 & 0,0 & 39,4 & 40,7 \\
\hline Urée. & 0,0 & 0,0 & 0,0 & $29, \mathrm{I}$ & 0,0 & 0,0 & 0,0 & 0,0 \\
\hline
\end{tabular}




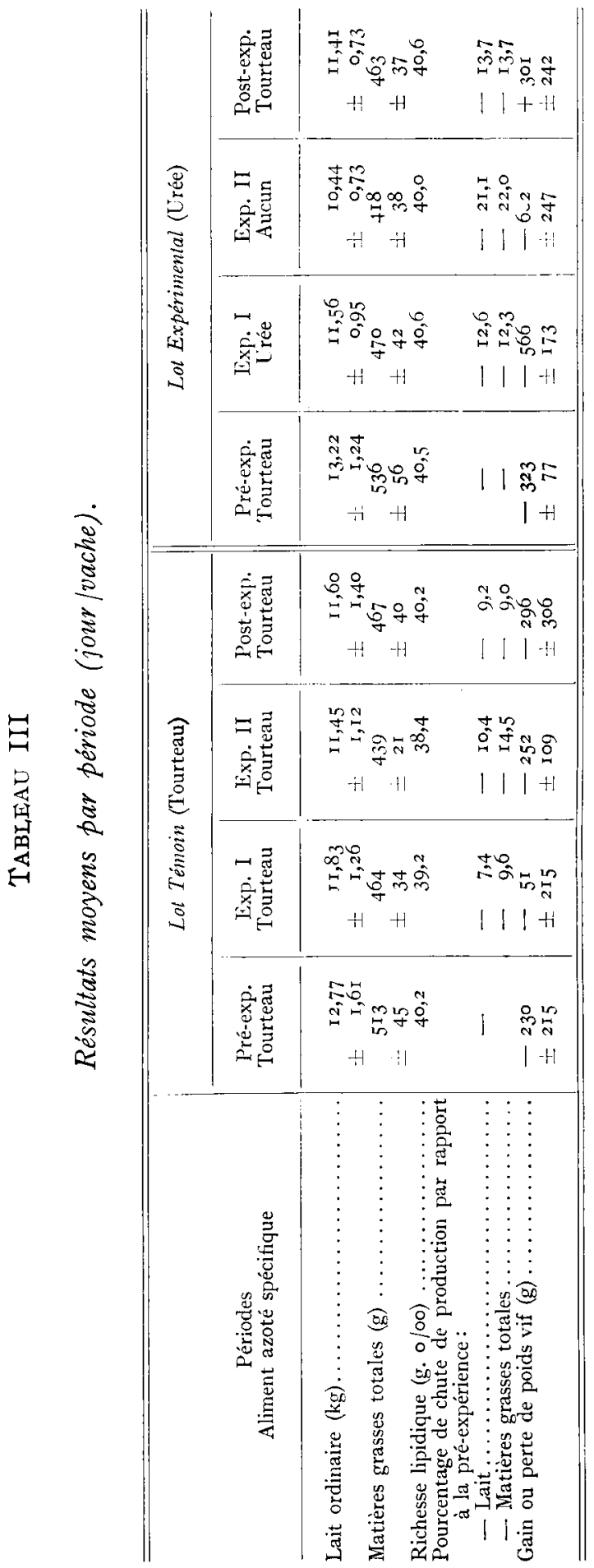




\section{B. - ÉVOLUTION DES SÉCRÉTIONS MAMMAIRES ET DU POIDS VIF}

Les résultats de production moyenne de lait et de graisse de lait, ainsi que les gains ou pertes de poids vif figurent dans le tableau III. Les mesures expérimentales détaillées sont représentées graphiquement (fig. I, 2, 3, 4).

\section{DISCUSSION DES RESULTATS}

La période préexpérimentale où les deux groupes de vaches ont été soumis au régime de référence renfermant les tourteaux, admise comme terme de comparaison, on constate que la substitution d'urée à ces der-

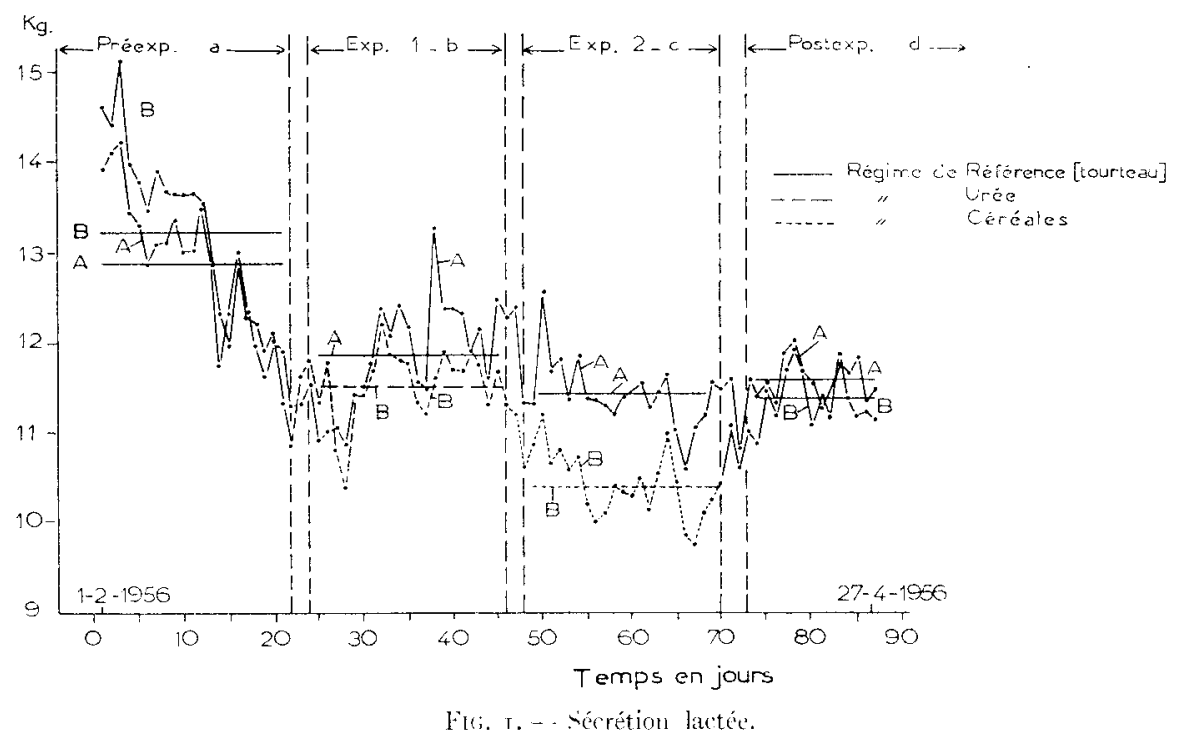

niers dans la ration du groupe $B$ (expérimental) pendant l'expérience I (b) entraîne une baisse journalière absolue du niveau moyen de production, de $-\mathrm{I}, 66 \mathrm{~kg}$ de lait et de $-66 \mathrm{~g}$ de graisses totales contre $-0,94 \mathrm{~kg}$ et - $49 \mathrm{~g}$ observée dans le groupe témoin (A) ; ce qui représente respectivement une chute de - I2,6\% et - I2,3\% au lieu de $-7,4 \%$ et $-9,6 \%$.

Avec le régime qui contient de l'urée à la place de tourteaux, la diminution de production est donc plus prononcée. I a suppression non compensée de cette source d'azote au cours de la contre-expérience (période c) qui a suivi, accentue davantage l'écart entre les groupes témoin et expérimental; par rapport à la préexpérience, les productions de lait 
et de graisse décroissent pendant cette période dans le premier groupe de - I,32 $\mathrm{kg}$ de lait et $-74 \mathrm{~g}$ de graisses totales soit de : - ro,4\% et - I $4,4 \%$; elles fléchissent dans le second, respectivement de $-2,78 \mathrm{~kg}$ et - II 8 g soit de - 2 I,I \% et - $22,0 \%$.

Les écarts dus à l'introduction ou à la suppression de l'urée dans le régime expérimental peuvent être interprétés soit par la méthode de

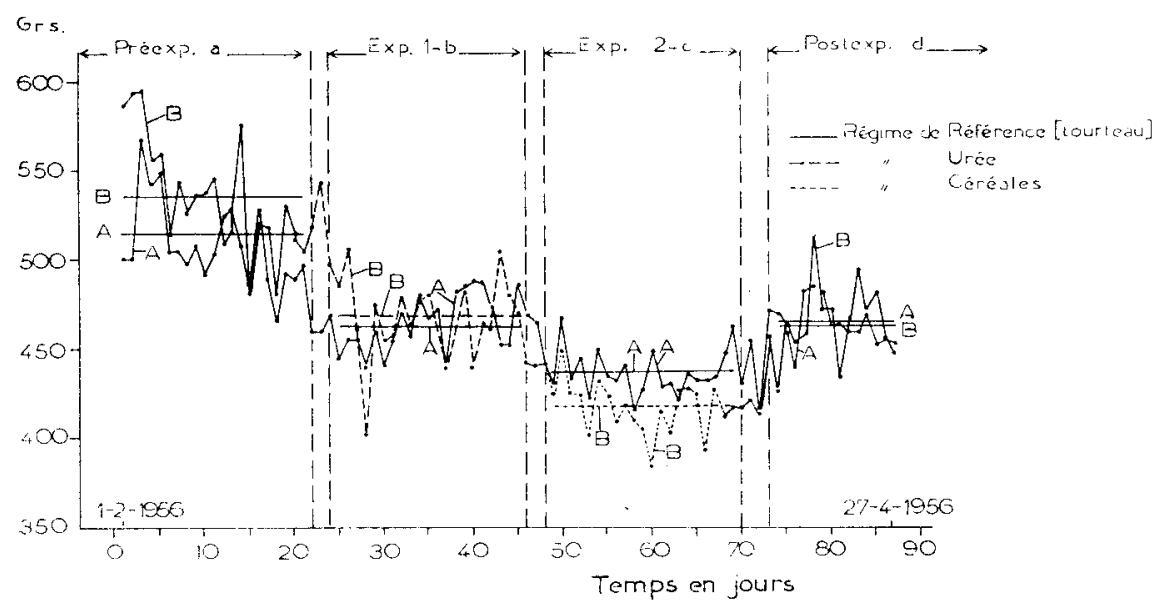

lisc. 2. . . Sécrétion lipidique totale.

"groupes comparables", soit par celles de "périodes successives" puisque 1'expérience a été conduite selon ces 2 techniques combinées.

\section{Io Interprétation des résultats par la méthode des groupes comparables.}

On sait qu'après son passage au maximum, la production metisuelle de lait d'une vache regresse d'un mois à l'autre selon une constante appelée coefficient de persistance. Dans nos expériences dont les périodes duraient 2 I jours, le terme coefficient de persistance exprime seulement le rapport entre la production d'une période donnée et celle de la période de référence (préexpérience) afin de pouvoir expliciter mathématiquement la réaction des animaux aux modifications de régime. La comparaison de ces coefficients pour la période b où les sujets expérimentaux recevaient de l'urée fait ressortir entre les 2 groupes une différence moyenne de $-5,2 \%$ pour le lait et de $-2,6 \%$ pour la matière grasse totale (voir tableau IV).

Ces écarts qui ne sont pas significatifs au seuil de probabilité de $5 \%$ (pour le lait $0,2>\mathrm{P}>0, \mathrm{I}$; pour les graisses $0,4>\mathrm{P}>0,3$ ) n'en indiquent pas moins (fig. I) que si le remplacement de l'azote de tourteau par celui d'urée déprime fort peu la synthèse lipidique, il 
n'assure pas, en revanche, le maintien à son niveau normal de la sécrétion lactée; en effet, tandis que la persistance de celle-ci paraît normale chez les sujets témoins $(0,927 \pm 0,020)$ chez les animaux expérimentaux, elle est nettement subnormale $(0,879 \pm 0$, org $)$ et les réactions individuelles, toutes dans le même sens, confirment ce phénomène. A la suite de la suppression de l'urée sans contrepartie, les écarts de production entre sujets expérimentaux et témoins sont plus importants et deviennent significatifs : ils atteignent - II, $3 \%$ pour le lait et $-8,7 \%$ pour les graisses $(0,02>\mathrm{P}>0$, oI pour le lait et $0,05>\mathrm{P}>0,02$ pour les graisses.

\section{TABI,EAU IV}

Coefficients individuels de persistance de sécrétion mammaire.

\begin{tabular}{|c|c|c|c|c|}
\hline \multirow{2}{*}{$\begin{array}{l}\text { Expérience I } \\
\text { (période b) }\end{array}$} & \multicolumn{2}{|c|}{ Sécrétion lactée } & \multicolumn{2}{|c|}{ Sécrétion lipidique totale } \\
\hline & Groupe A & Groupe $\mathrm{B}$ & Groupe A & Groupe I3 \\
\hline Rapport interjériodique & $\frac{b(\text { tourteau })}{a(\text { tourteau })}$ & $\frac{b(\text { uróe })}{a(\text { tourteau })}$ & $\frac{b}{a} \frac{(\text { tourteau })}{\text { (tourteau })}$ & $\frac{b \text { (urée) }}{a \text { (urée) }}$ \\
\hline 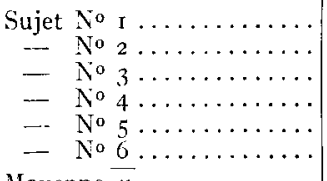 & $\begin{array}{l}0,910 \\
0,869 \\
0,936 \\
0,894 \\
\text { I,0 } 3 \\
0,040\end{array}$ & $\begin{array}{l}0,945 \\
0,879 \\
0,802 \\
0,899 \\
0,875 \\
0,87 \mathrm{I}\end{array}$ & $\begin{array}{l}0,025 \\
0,851 \\
0,894 \\
0,910 \\
0,963 \\
0,908\end{array}$ & $\begin{array}{l}0,946 \\
0,8,34 \\
0,861 \\
0,927 \\
0,8,30 \\
0,920\end{array}$ \\
\hline Moyenne $\bar{x} \ldots \ldots \ldots \ldots$ & $0,927=0,020$ & $0,879 \div 0,019$ & $\begin{array}{r}0,909: \pm 0,015 \\
-\end{array}$ & $0,886 \pm 0,02 \mathrm{I}$ \\
\hline $\begin{array}{l}\text { Différence en défaveur du } \\
\text { régime expérimental.... }\end{array}$ & $\begin{array}{r}\bar{x}_{\mathrm{B}}-\bar{x}_{\mathrm{A}}=0,87 \\
\text { soit }\end{array}$ & $\begin{array}{l}-0,927=-0,048 \\
5,2 \%\end{array}$ & 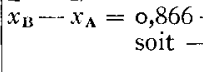 & $\begin{array}{l}0,909-0,023 \\
2,6 \%\end{array}$ \\
\hline $\begin{array}{l}\text { Signification statistique de } \\
\quad \text { la différence } . . . \ldots \ldots \ldots\end{array}$ & $\mathrm{t}=\mathrm{I}, 73^{\circ}$ & $2>\mathrm{I}>0, \mathrm{I}$ & $\mathrm{t}=0,897 ; 0$ & $>\mathrm{P}>0,3$ \\
\hline $\begin{array}{l}\text { Expérience II } \\
\text { (période c) }\end{array}$ & $\frac{c \text { (tourteau) }}{a \text { (tourteau) }}$ & $\begin{array}{c}\text { (Suppression non } \\
\text { compensée d'urée) } \\
\frac{c \text { (pas d'urée) }}{a \text { (tourteau) }}\end{array}$ & $\frac{c(\text { tourteau })}{a \text { (tourteau) }}$ & $\frac{c(\text { pas d urce })}{a \text { (tourteau })}$ \\
\hline 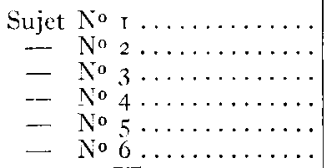 & $\begin{array}{l}0,9 \text { I I } \\
0,814 \\
0,919 \\
0,937 \\
0.958 \\
0,864\end{array}$ & $\begin{array}{l}0,853 \\
0,797 \\
0,701 \\
0,782 \\
0,785 \\
0,877\end{array}$ & $\begin{array}{l}0,945 \\
0,764 \\
0,879 \\
0,863 \\
0,881 \\
0,838\end{array}$ & $\begin{array}{l}0,84 \mathrm{I} \\
0,757 \\
0,7,57 \\
0,785 \\
0,76 \mathrm{r} \\
0,8 \mathrm{I} 9\end{array}$ \\
\hline Moyenne $\bar{x} \ldots \ldots \ldots \ldots$ & $0,901 \pm 0,022$ & $0,799 \pm 0,025$ & $0,862 \pm 0,024$ & $0,787 \geq 0,015$ \\
\hline $\begin{array}{l}\text { Différence en défaveur du } \\
\text { régime expérimental... }\end{array}$ & $\begin{array}{c}\bar{x}_{B}-\bar{x}_{A}=0,799 \\
\text { soit }\end{array}$ & $\begin{array}{l}-0,90 \mathrm{I} \\
\mathrm{II}, 3 \%\end{array}$ & $\begin{aligned} \bar{x}_{\mathrm{B}}-\bar{x}_{\Lambda} & =0,787 \\
& \text { soit }\end{aligned}$ & $\begin{array}{l}-0,868=-0,075 \\
8,7 \%\end{array}$ \\
\hline $\begin{array}{l}\text { Signification statistique de } \\
\text { la différence } \ldots \ldots \ldots \ldots\end{array}$ & $t=3,095 ; 0$ & $02>\mathrm{I}^{\prime}>0, \mathrm{OI}$ & $\mathrm{t}=2,643 ; 0$, & $>P>0,02$ \\
\hline
\end{tabular}

Tels quels, ces résultats n'étant pas dégagés de l'influence de l'évolution physiologique de la lactation, leur analyse par cette méthode est susceptible de masquer l'action effective du traitement expérimental et fausser de la sorte les conclusions. 


\section{$2^{\circ}$ Interprétation par la méthode des périodes successives.}

La technique de "périodes successives" qui élimine cette source d'erreurs et possède l'avantage de mettre en évidence le comportement d'un même sujet en présence d'une modification des conditions alimentaires, rend plus précise la mesure de l'action d'un facteur nutritionnel.

I'application de l'ajustement linéaire (valable pour des périodes suffisamment courtes) aux sécrétions mammaires enregistrées au cours de la pré- et post-expérience, permet d'en dédurire leur régression physiologique naturelle chez chaque individu. $\mathrm{I}_{4}$ connaissance de cette droite d'ajustement autorise le calcul par intrapolation des quantités théoriques de lait et de matière grasse qu'il aurait été donné d'observer durant les périodes intercalaires à traitement expérimental (incorporation ou suppression d'urée), si les conditions de nutrition étaient restées inchangées ; il est admis que l'écart entre ces données calculées et celles réellement mesurées traduit 1'action du traitement etudié.

TABLEAU $\mathrm{V}$

Comparaisons entre les productions théoriques $(t)$ et réelles $(r)$ du groupe expérimental $(B)$.

Expérience I

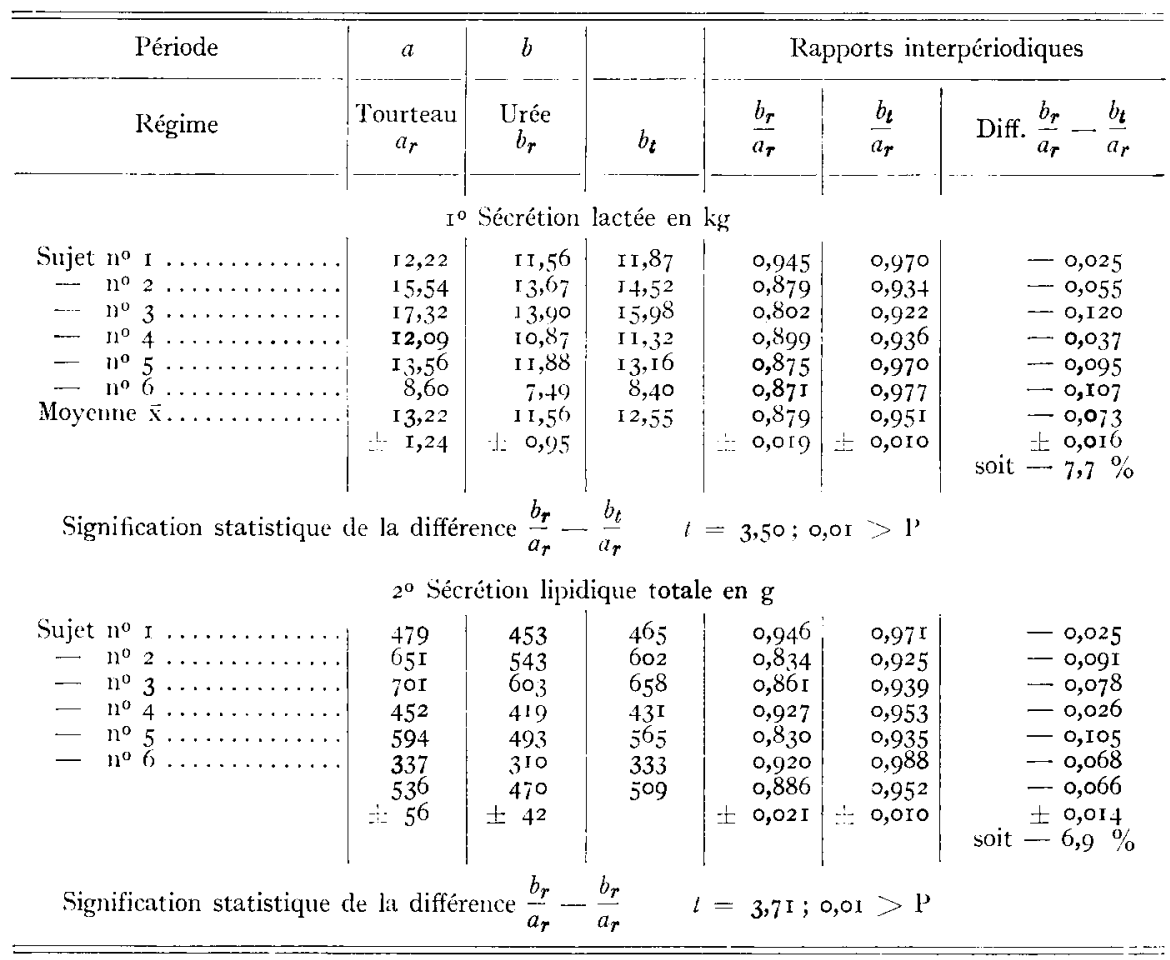


Les rapports interpériodiques des productions théoriquement escomptées à partir de ces calculs et leur comparaison avec ceux calculés sur la base des données réelles chez les animaux ayant subi le régime d'expérience sont consignés dans les tableaux V et VI.

\section{TABLEAU VI}

Comparaison entre les productions théoriques $(t)$ et réelles $(r)$ du groupe expérimental $(B)$.

Expérience II

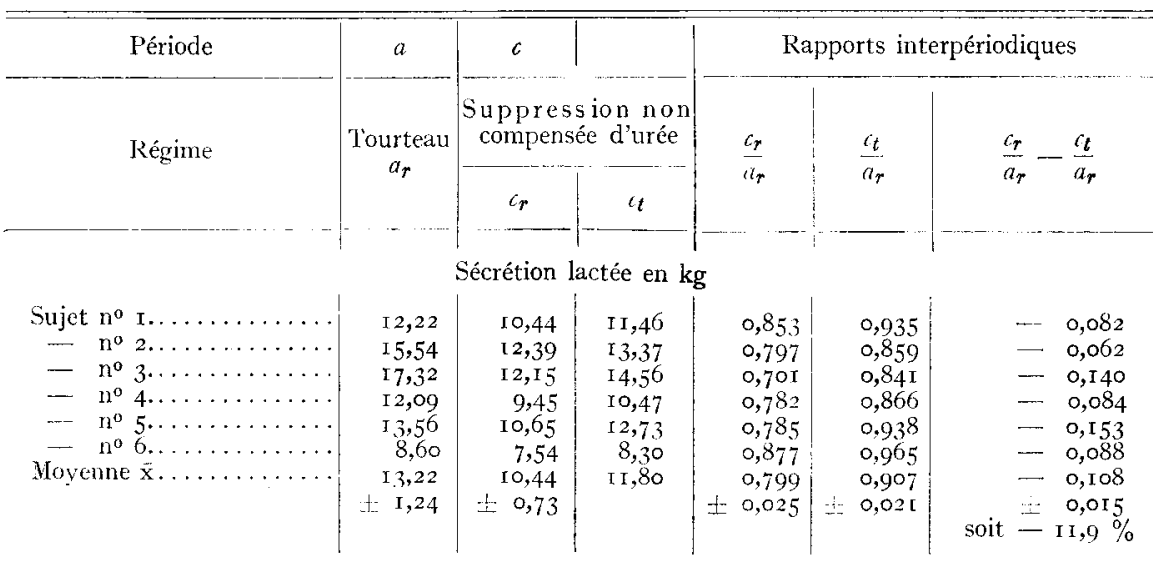

Signification statistique de la différence $\frac{c_{r}}{a_{r}}-\frac{c_{t}}{a_{r}} \quad t=3,28 ; 0,0 \mathrm{I}>\mathrm{P}$

Sécrétion lipidique totale en $\mathrm{g}$

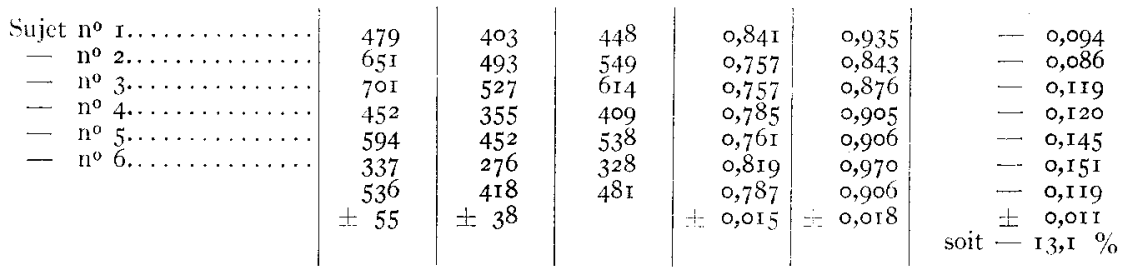

Signification statistique de la différence $\frac{c_{r}}{a_{r}}-\frac{c_{t}}{a_{r}} \quad t=5,15 ;$ o,or $>\mathrm{P}$

Ils montrent que les quantités individuelles de lait et de matière grasse sont significativement plus faibles lorsque l'azote uréique remplace celui de tourteaux $(\mathrm{P}<0, \mathrm{OI})$. Toutefois, les différences sont bien plus élevées et hautement significatives à la suite de la suppression non compensée de cette fraction azotée : par rapport à la théorie, la substitution d'urée aux tourteaux occasionne une diminution moyenne de $-7,7 \%$ de lait et de $-6,9 \%$ de graisse de lait; son élimination entraîne des chutes respectives une de - II,9\% et - I3,I \% .

A noter que l'introduction ou la suppression d'urée n'a modifié en rien 
la richesse lipidique des laits, (fig. 3) qui s'est maintenue remarquablement constante et conforme à ce que la théorie laissait prévoir. L'action de l'urée parait donc s'exercer exclusivement sur le volume de la sécrétion lactée. Et il n'est pas moins curieux de relever, pour les périodes correspondantes, une légère réduction du taux butyreux dans le groupe témoin (39,2 et 38,4 contre 40,2 durant les pré- et post-expériences) apparemment inexplicable puisque les conditions alimentaires de ce groupe sont restées pratiquement inchangées tout au long de l'expérience. On pourrait évidenument supposer une influence négative prolongée des très basses températures qui ont régné à ce moment et auxquelles l'homéotherme réagit comme on le sait $(24,25,26)$ par une mobilisation et une oxydation accélérée des graisses de l'organisme phénomènes qui se seraient effectués aux

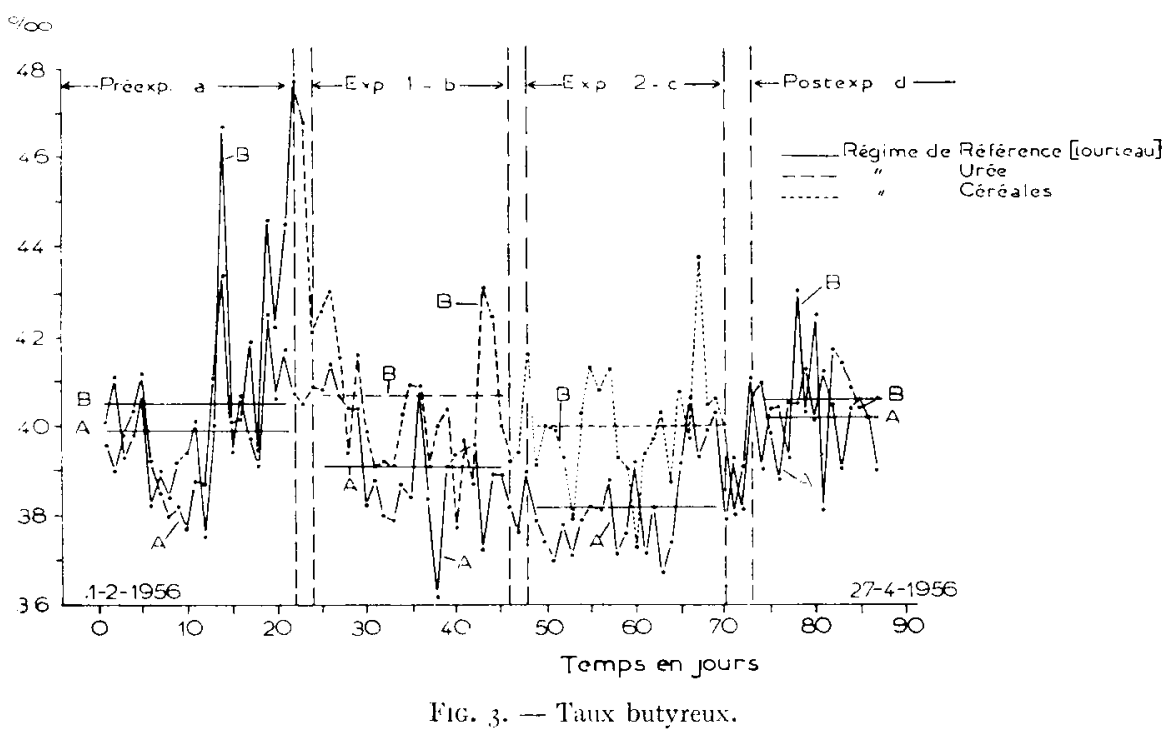

dépens de la synthèse lipidique; l'effet spécifiquement favorable de l'urée sur l'acétogénèse, démontrée par des observations in vitro (I8) aurait, par contre, exercé une action d'épargne dans le cas des sujets ayant ingéré cette substance; cette influence aurait continuée durant la période suivante, même après suppression du produit en question, par suite des modifications intervenues dans la microflore des réservoirs gastriques, mais semblable explication n'est formulée qu'à titre d'hypothèse.

D'une manière générale, il n'est pas impossible, comme il a déjà été dit, que l'influence du froid ait interféré avec celle de l'urée rendant ainsi moins nette la réponse des sujets du groupe $\mathrm{B}$ à ce traitement, comme le suggèrerait le léger accroissement des sécrétions mammaires observées dans le groupe témoin (A) au cours de la post-expérience après retour à la température normale. 
Le fait qu'après la suppression d'urée, la chute absolue des sécrétions mammaires s'amplifie nettement, vient à l'appui de la thèse favorable à l'urée, confirmée tout récemment encore par certains auteurs (I3, I4, I5, I6, I7). I1 n'en reste pas moins vrai que notre analyse du comportement des mêmes sujets soumis successivement aux divers régimes alimentaires, telle qu'elle vient d'etre faite (tableaux V et VI) indique sans conteste une utilisation défectueuse de l'urée aux fins d'une couverture des besoins protidiques d'entretien et de lactation chez la vache.

La vraisemblance de cette hypothèse se trouve corroborée par un phénomène d'un autre ordre (tableau III, fig. 4) ; l'administration d'urée

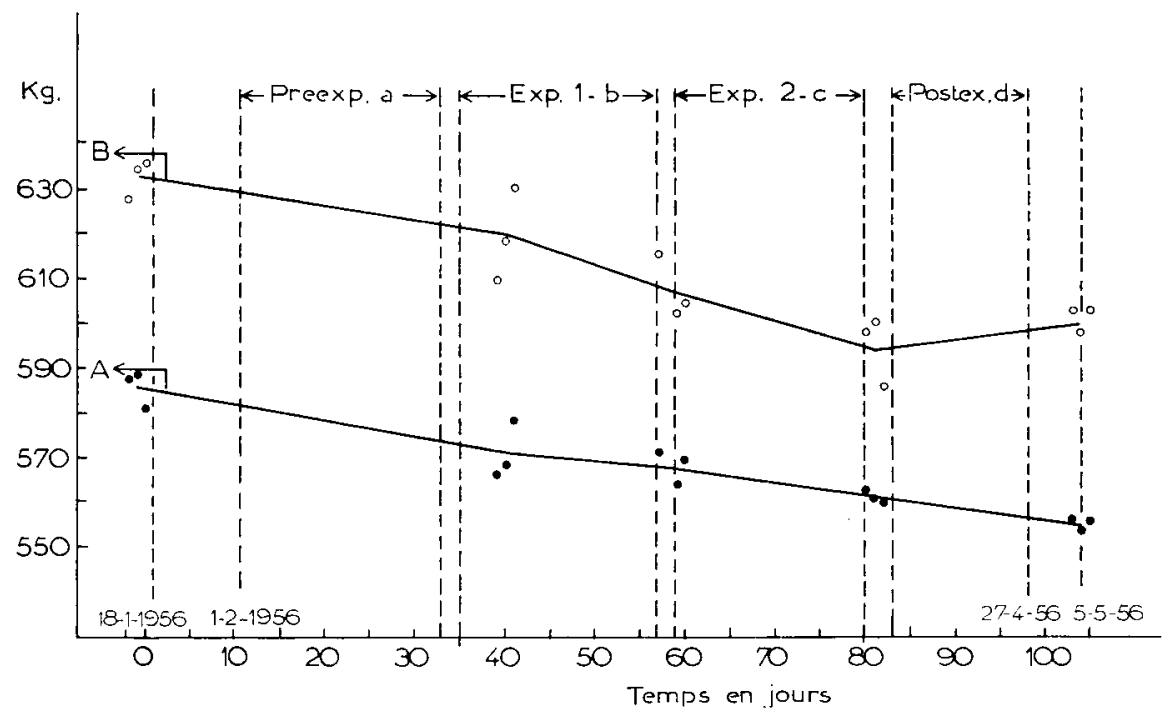

FIG. 4. - Evolution du poids corporel moyen.

s'accompagne d'un amaigrissement significatif des aninaux traités (-566 \pm I73 g par jour contre $5 \mathrm{I} \pm 2$ I $5 \mathrm{~g}$ pour les non traités), nonobstant un apport d'énergie dépassant les besoins de $+4,6 \%$ (tableau I). La réalité de cet amaigrissement que la suppression d'urée intensifie $(-602 \pm 247 \mathrm{~g})$ frappe d'autant plus que le retour de ces mêmes vaches au régime de références (post-expérience) a pour conséquence un gain appréciable de poids vif $(+301 \pm 242$ g.). Une observation analogue (non significative il est vrai) contemporaine de la nôtre est, d'ailleurs, rapportée par Rust et coll. (Ig) qui enregistrent également une chute de la production des vaches auxquelles ils ont donné de l'urée; il faut signaler que la plupart des auteurs n'ont pas étudié l'effet de l'urée sous cet aspect.

Dans nos conditions expérimentales, les baisses simultanées du 
niveau des sécrétions mammaires et du poids corporel témoignent indubitablement d'une moindre efficacité de l'azote de l'urée par rapport aux tourteaux pour la couverture des besoins de la vache laitière; elles laissent en particulier supposer que, par suite de la médiocrité des protides alimentaires du régime renfermant cette source azotée, les animaux du groupe expérimental aient dî faire des emprunts à leurs protéines tissulaires afin de faire face aux exigences prioritaires de la lactation.

L'éventualité d'une influence de nature énergétique stimulant davantage les activités mammaires des animaux consommant des tourteaux que celles des sujets alimentés avec de l'urée paraît improbable. La faible valeur de l'urée telle que la fait paraitre l'ensemble de nos mesures biologiques ne saurait, en effet, être imputée aux apports d'énergie qui, dans les deux lots, excédaient largement les besoins théoriques durant toutes les périodes, excepté la pré-expérience.

On pourrait supposer, d'autre part, que l'insuffisance des régimes tant témoin qu'expérimental en lipides alimentaires ait exercé une action de carence sur les sécrétions mammaires malgré les rations copieuses de fourrages cellulosiques consommées (I2) ; s'il en avait été ainsi, cette déficience aurait dû déprimer la production de tous les animaux mais beaucoup moins celle des sujets ayant ingéré de l'urée, substance qui accroît, comme on le sait, les disponibilités en acides gras volatils précurseurs de graisse de lait (I8).

Par ailleurs, la richesse des rations en glucides facilement utilisables par les microorganismes du rumen et présents sous forme de glucides de betteraves $I / 2$ sucrières, d'orge et d'avoine ne pouvait que favoriser la synthèse à partir d'urée des protéines bactériennes profitables à la formation de protides de lait.

Il est donc logique de rechercher l'origine du fléchissement de la production et du poids vif des animatx traités à l'urée dans les niveaux et la qualité de l'azote des régimes en présence, que nous examinerons attentivement. Si l'on défalque, de l'azote total digestible ingéré, les dépenses d'entretien que l'on peut raisonnablement estimer à Io g par ioo $\mathrm{kg}$ de poids vif (cf. Amrsby (20), Morrison. (23) Moliganad (22)) on constate que les groupes témoin et expérimental disposaient par $\mathrm{kg}$ de lait à 4 p. roo au cours des diverses périodes des quantités d'azote (Kjeldahl) indiquées au tableau VII.

Ces quantités assuraient par $\mathrm{kg}$ de lait à $4 \mathrm{p}$. Ioo produit au groupe témoin $83 \mathrm{~g}$ au moins et $100,9 \mathrm{~g}$ au plus de protides totaux digestibles ; en comparaison avec les normes exprimées en équivalents protéiques, ces niveaux n'ont, de prime abord, rien d'excessif; elles le paraissent fortement si on les confronte avec les standards de MORrison (2x), qui considère un apport de $49 \mathrm{~g}$ comme un maximum. 
TABLEAU VII

Disponibilités protidiques effectives par $k g$ de lait à 4 p. 100 (moyennes pondérées).

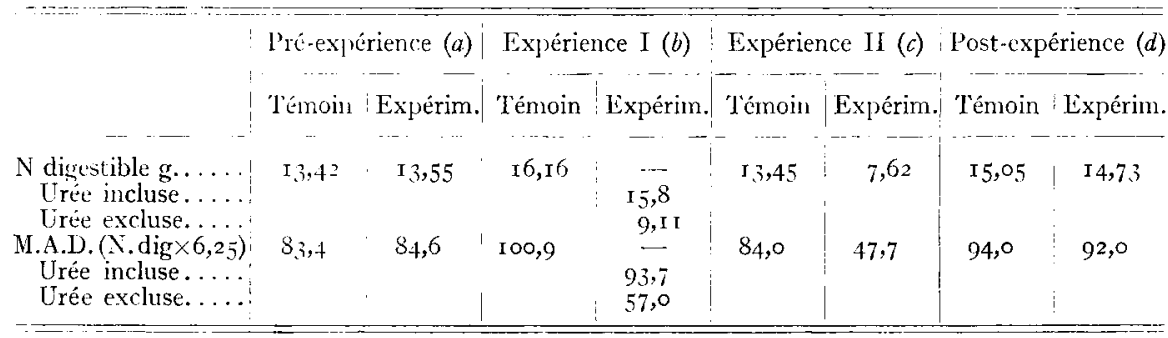

Pour le groupe expérimental B, on note pendant les pré- et post-expériences des niveaux azotés proches de ceux du témoin. Durantl'expérienceI, compte tenu de l'apport azoté dû̀ à l'urée, ce groupe disposait de 93,7 $\mathrm{g}$ de matières azotées digestibles par $\mathrm{kg}$ de lait, mais de $57 \mathrm{~g}$ seulement si on le néglige. La confrontation de ces quantités avec les niveaux correspondants de production montre que les derniers diminuent davantage dans le groupe expérimental que dans le témoin (tableaux IV, V, VI), malgré un apport azoté très voisin (93,7 $\mathrm{g}$ contre Ioo,9 g). Considérant, de plus, les pertes de poids vif enregistrées parallèlement, cela donne à supposer que :

- la fraction azotée provenant de l'urée $(93,7$ - 57,0 soit $36,7 \mathrm{~g})$ est qualitativement moins appropriée au maintien de la lactation que celle des tourteaux, puisque les autres sources d'azote des deux régimes sont exactement les mêmes;

- un niveau de $57 \mathrm{~g}$ de matières azotées digestibles (en supposant la non utilisation de $\mathrm{N}$ uréique) parait être en-dessous du seuil minimum probablement compatible avec un niveau normal de production laitière ; ce qui confirmerait les observations de DELAGE et Françors (27) dont les animaux ont accusé une baisse très nette de leur sécrétion de lait alors qu'ils ne recevaient pour la satisfaction de leur besoin de production qu'un apport de bo $\mathrm{g}$ de matières azotées digestibles par $\mathrm{kg}$ de lait.

Au cours de la contre-expérience (période c) où les sujets du groupe expérimental étaient soumis intentionnellement au niveau azoté subnormal (suppression non compensée de l'urée), les disponibilités par kg de lait sont tombées à $47,7 \mathrm{~g}$; il s'en est suivi une diminution très significative des sécrétions mammaires et une chute du poids vif encore plus forte qu'au cours de la période qui l'a précédée. Ces phénomènes constituent un argument qui témoigne indiscutablement:

- de l'insuffisance du niveau azoté ci-dessus, considéré pourtant par MORRISON comme un maximum ; 
- d'un taux d'utilisation sinon nul, du moins très bas cle l'urée, fait qui confirme les études in vitro rapportées ailleurs (23).

Incidemment, l'ensemble de ces constatations renforce l'opinion que pour le type de régime alimentaire de pratique courante en Irance, utilisé dans cette expérience et comportant une grosse part d'azote non protéique, les apports de matières azotées totales digestibles par $\mathrm{kg}$ de lait à $4 \mathrm{p}$. Ioo doivent être supérieurs à $57 \mathrm{~g}$.

\section{RESUMÉ ET CONCLUSIONS}

Une expérience a été conduite selon la technique combinée de groupes comparables et de périodes successives avec 12 vaches normandes en lactation afin de comparer l'efficacité de l'azote d'urée à celui de tourteaux de lin et d'arachide pour la production de lait et de graisses de lait. Les régimes de référence (tourteaux) et d'expérience (urée) étaient équiazotés. Au cours d'une première expérience, l'urée fournissait 29, I p. Ioo de l'azote digestible total du régime expérimental renfermant en sus des céréales, des betteraves, des balles de blé et du foin. Ces derniers éléments procuraient 59,7 p. Ioo des protides totaux digestibles ingérés.

Durant la contre-expérience qui a suivi, l'urée a été supprimée sans compensation aucune d'azote pour le groupe expérimental.

Il a été constaté que:

- la substitution d'azote uréique à celui de tourteaux d'arachide et de lin entraînait une diminution appréciable des productions de lait, de graisses de lait et de poids vif;

- la suppression non compensée de l'urée accentuait le phénomène de baisse, qui est devenu hautement significatif quelle que soit la méthode d'interpretation des résultats.

Il s'ensuit que 1'azote uréique est incapable d'assurer aux fins de lactation un équilibre azoté optimum et comparable à celui que procurent les protides de tourteaux de lin et d'arachide ; les pertes de poids corporel consécutives à l'ingestion d'urée font présumer que l'animal ferait emprunt à ses protéines tissulaires pour suppléer à l'efficacité médiocre des régimes comportant de l'urée, qui ne serait que très partiellement employée à la couverture des besoins mixtes d'entretien et de lactation chez la vache.

Reçu pour publication le 27-2-58.

\section{REFFERENCES BIBLIOGRAPHIQUES}

(I) REID (J. T.). - J. Dairy Sci., 36, 955, I953.

(2) Ferrando (R.). - Méd. Vét., 107, 96, I956.

(3) Voltz (W.), Dietrich (W.), Jantzon (H.). - Biochem. Zeit., 130, 323 , I922. 
(4) Richarsen (A.), Brinkmann (O.). - Fuhl. Landw. Zeit., 71, 325, I922.

(5) Owen (E. G.), Smith (J. A. B.), Wright (N. G.). - Biochem. J., 37, 44, I943.

(6) Rupei (L. W.), Bohstedt (G.), Hart (E. B.). - J. Dairy Sc., 26, 647, I943.

(7) Wiriter' (E. M.), Henke (L. A.), Maruyama (C.). - J. Dairy Sc., 29, $629,1946$.

(8) Hastings (W. H.). - J. Dairy Sci., 2\%, I0I5, 1944.

(9) Thompson (N. R.), Graf (G. C.), Eheart (J. F.), Holdaway (C. V.). J. Dairy Sci., 35, IOIO, I952.

(io) Archibali (J. G.). - Mass. Agr. Exp. St. Bull. $1^{\circ} 406$, I943.

(II) Bartlett (S.), Blaxter (K. L.). - J. Agri. Sci., 3\%, 32, I947.

(I2) Bai,CH (C. C.), BAlCh (D. A.), BartietT (S.), Cox (C. P.), Rowiand (S. J.). - J. Dairy Research, 19, 39, I952.

(I3) Bainter (K.), Barabas (E.), Moxostori (I.). -- In Dairy Sci. Abst., 18,8 I5, I $95^{6}$.

(I4) Lassitek (C. A.), Seath (D. M.), Hamblin (F. B.), Adams (B. M.), BASTIN (G. M.). -. In Dairy Sci. Abst., 18, 815, I956.

(I5) Davis (C. I.), Lassiter (C. A.), Slitih (I). M.), Rust (S. V.). - J. Anim. Sci., 15, 5I5, I956.

(i6) Otagaki (K. K.), Wayman (O.), Morita (K.). - I'roc. W'. Div. Amer. Dairy Sci. Ass., p. 55, I956.

(I7) Matsun (T.), Ushizima (T.). - In Dairy Sci. Abst., 19, 709, 1957.

(I8) ZEITER (S. Z.), LEROY (F.). - Ann. Zoot., \%, no 3 ; i 73, $195^{8}$.

(I9) Rust (J. V.), Lassiter (C. A.), Iavis (C.), Brown (L. D.), Seath (D. M.). - J. Anim. Sci., 15, I133, I956.

(20) Armsby (H. P.). - The nutrition of farm animals (Mac Millan, New York, I9I7).

(2I) Morrison (F. B.). - Feeds and Feedings (Ithaca, New York), I950.

(22) MoligaARD (H.). - Grundzüge der Ernahrungsphysiologie der Haustiere (Parey, Berlin), I93I.

(23) ZELTER (S. Z.), LEROY (F.). - Ann. Zoot., n, no 3; i85, 1958.

(24) Schaeffier (G. S.), Pollack (A.) - - C. R. Soc. Biol., 12\%, i76, I938.

(25) Poldack (A).-C.R. Soc. Biol., 130, I 49, I939.

(26) Clément (G.). - a) Ann. Nutr. Alim., 4, 295, I950-b) Arch. Sci. Physiol., 4, 5, I95o et 5, I69 I95I.

(27) Delage (J.), Françors (A.). - Communication Ve Congrès International de Zootechnie, Paris, I949.

Nous tenons à exprimer nos vifs remerciements à M. SALger, Ingénieur en Chef. Directeur des Services Agricoles de Seine-Maritime et à M. Faucon, Directeur du Centre de rééducation d'Aumale, qui ont bien voulu mettre a notre disposition les animaux de ce centre. 